Continuous phase-shift lithography with a roll-type mask and application to transparent conductor fabrication

This article has been downloaded from IOPscience. Please scroll down to see the full text article.

2012 Nanotechnology 23344008

(http://iopscience.iop.org/0957-4484/23/34/344008)

View the table of contents for this issue, or go to the journal homepage for more

Download details:

IP Address: 141.211.173.82

The article was downloaded on 25/06/2013 at 20:36

Please note that terms and conditions apply. 


\title{
Continuous phase-shift lithography with a roll-type mask and application to transparent conductor fabrication
}

\author{
Moon Kyu Kwak ${ }^{1,2}$, Jong G Ok ${ }^{3}$, Jae Yong Lee ${ }^{1}$ and L Jay Guo ${ }^{1,3}$ \\ ${ }^{1}$ Department of Electrical Engineering and Computer Science, University of Michigan, Ann Arbor, \\ MI 48109, USA \\ ${ }^{2}$ School of Mechanical Engineering, Kyungpook National University, Daegu, 702-701, \\ Republic of Korea \\ ${ }^{3}$ Department of Mechanical Engineering, University of Michigan, Ann Arbor, MI 48109, USA \\ E-mail: guo@eecs.umich.edu
}

Received 20 February 2012, in final form 21 March 2012

Published 10 August 2012

Online at stacks.iop.org/Nano/23/344008

\begin{abstract}
We report the development of a near-field optical nanolithography method using a roll-type phase-shift mask. Sub-wavelength resolution is achieved using near-field exposure of photoresist through a cylindrical phase mask, allowing dynamic and high throughput continuous patterning. As an application, we present the fabrication of a transparent electrode in the form of a metallic wire grid by using the roller-based optical lithography method. To fabricate a mesh-type metal pattern, a specific phase-shift mask was designed and critical experimental parameters were also studied. As a result, a transparent conductor with suitable properties was achieved with a recently built cylindrical phase-shift lithography prototype designed to pattern on $100 \mathrm{~mm}^{2}$ of substrate area.
\end{abstract}

(Some figures may appear in colour only in the online journal)

\section{Introduction}

There is an increasing demand for large area, low cost nanofabrication techniques for many applications. These applications include nanostructured self-cleaning surfaces [1,2], biomimetic dry adhesives [3], nanopatterned light-trapping layers and nanostructured absorbers for photovoltaic devices $[4,5]$, wire grid polarizers for display devices [6, 7], and optical metamaterials [8, 9], to name an important few. Current available nanopatterning and fabrication techniques are still unable to meet the required performance, fabrication speed, and cost criteria for such large area patterning applications. To address this need, the roll to roll nanoimprinting (R2RNIL) technique was introduced [10]. R2RNIL demonstrated polymer patterns down to $70 \mathrm{~nm}$ feature size in a continuous process and was also shown to be suitable for device applications [11]. If the imprinted polymer structure is the final product, R2RNIL could be one of the best methods. However, in R2RNIL the patterning speed is limited by the imprinting and demolding process. Also the patterns created by the R2RNIL process always involve a residual layer, which needs to be very thin compared with the pattern depth and needs to be removed by a plasma etching process if the imprinted polymer pattern is to be used as a mask. Consequently this will affect the mass production speed and the cost of the process.

Recently a continuous near-field optical nanolithography method was introduced using a roll-type phase-shift mask [12]. The technique is based on phase lithography using an elastomeric stamp that was introduced a decade ago and has been developed over the years [13-15]. The new roller phase lithography technique combines some of the best features of photolithography, soft lithography, and continuous roll to roll/plate patterning technologies [12]. We demonstrate that the technique can be scaled for a continuous and high throughput nanomanufacturing process. In contrast to previous approaches that used PDMS elastomer as phase masks $[12,13,15]$, our roller phase mask is made use of relatively rigid polymer, which helps to maintain the pattern height on the phase mask during the process, which is the most 


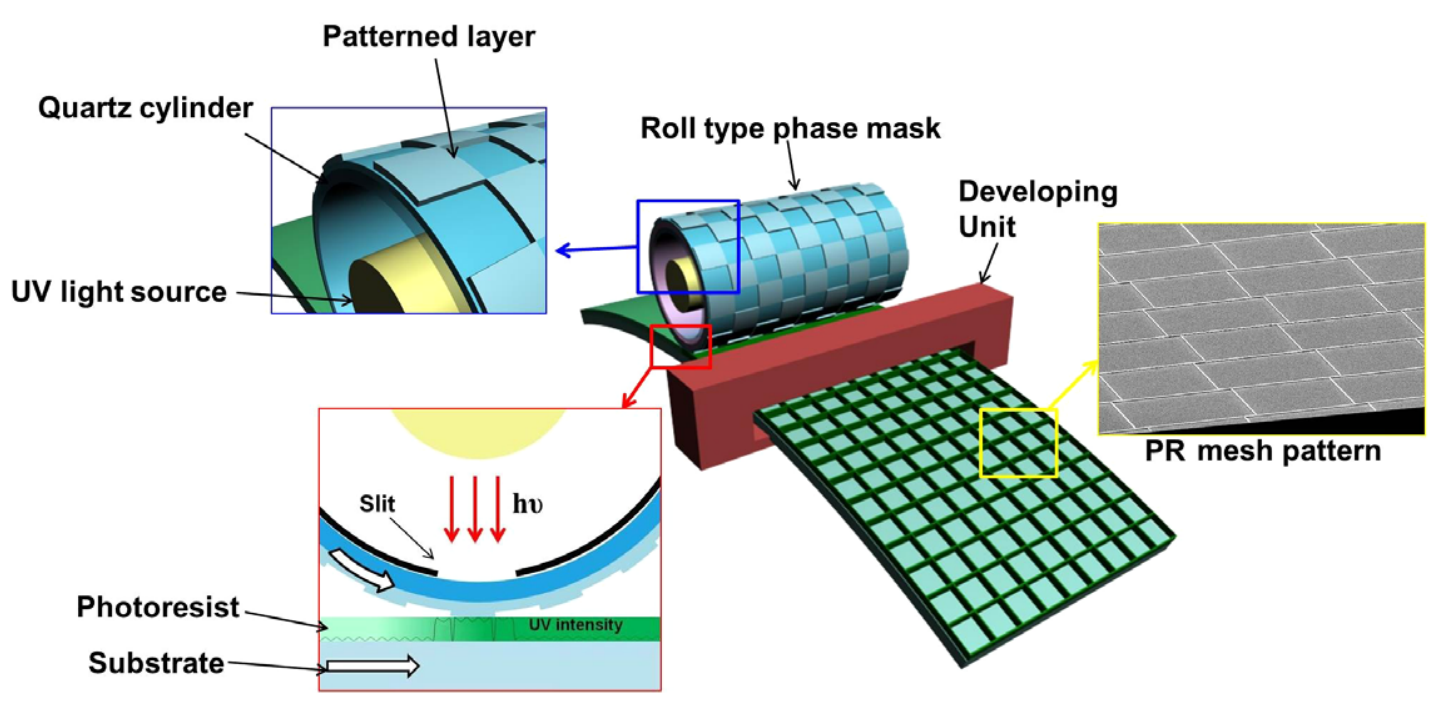

Figure 1. Schematic of roll-type phase lithography to make sub-micron mesh patterns for a transparent metal electrode. The SEM image represents a metal mesh pattern after lithography and dry etching.

critical parameter during phase lithography. As a result, we have significantly increased the reproducibility of the process, which is known to be a limiting factor reported previously. With this technique, various shaped large area patterns, including circular or bent patterns and straight line patterns, were successfully fabricated. We also report the fabrication of a metallic wire grid based transparent conductor by using this lithographic technique, combined with a dry etching process. $\mathrm{Al}$ was used for metallic transparent conductor owing to its low cost and ease of fabrication.

\section{Experimental details}

\subsection{Roller phase lithography setup and process}

Figure 1 shows a schematic diagram of the experimental set up for roller phase lithography. The set up consists of a roll-type phase mask, an $x y z$-stage, a substrate moving unit, and a UV exposing unit. The phase mask was fabricated by procedures described below. For the roll-type phase mask, a $90 \mathrm{~mm}$ diameter quartz cylinder tube was used to support the flexible phase mask. A remodeled UV light source (CS2010, Thorlabs) was installed inside the quartz cylinder. The wavelength was fixed on $365 \mathrm{~nm}$ and the intensity was regulated by the controller in the range of $0-200 \mathrm{~mJ} \mathrm{~cm}^{-2}$. A collimating lens is used to produce uniform UV light illumination, which exposes the resist through a slit. The substrate used for making the transparent electrode is Al-coated PET. Photoresist (S1805, Shipley) was spin-coated on the Al layer at $4000 \mathrm{rpm}$ for $30 \mathrm{~s}$. After baking at $95^{\circ}$ for $1 \mathrm{~min}$, PR-coated Al substrate was put on the moving stage and was brought into contact with the roll phase mask. An applied force between the quartz tube and the moving stage ensures a tight contact, and the linear motion of the stage also rotates the quartz tube carrying the roll phase mask by the friction force. Therefore a continuous patterning system was realized. An exposed sample with suitable intensity was able to be developed in about $1 \mathrm{~min}$ with the developer (MF-319, Shipley)

\subsection{Fabrication of a roll-type PUA phase mask}

A small amount $(0.1-0.5 \mathrm{ml})$ of a UV curable polyurethaneacrylate (PUA; Minuta Tech.) [16] pre-polymer was drop-casted on a pre-fabricated silicon master and a supporting PET film was carefully placed on top of the PUA surface to make conformal contact. To cure the PUA, the film mold was exposed to UV $(250-400 \mathrm{~nm})$ for $10 \mathrm{~s}$ at an intensity of $100 \mathrm{~mW} \mathrm{~cm}^{-2}$. After UV curing, the PUA mold was peeled off from the master and wrapped around the quartz tube via an adhesion layer that completes the fabrication of the roll-type phase mask.

\subsection{Al dry etching process}

$\mathrm{Al}$ was used to make the transparent conductors in the form of $\mathrm{Al}$ mesh. For better dimensional control in the sub-micron scale, dry etching of $\mathrm{Al}$ was used rather than wet etching. The $\mathrm{Al}$ etching was performed using $\mathrm{BCl}_{3} / \mathrm{Cl}_{2}$ chemistry in an inductively coupled plasma tool. The $\mathrm{Al}$ layer etching process consists two steps; in the first step native aluminum oxide was etched using $\mathrm{BCl}_{3}, 40 \mathrm{sccm}$ for $5 \mathrm{~s}$ with a RF power of $100 \mathrm{~W}$ and working pressure of $10 \mathrm{mT}$. The second step involved etching an aluminum layer by changing $\mathrm{BCl}_{3}$ to $20 \mathrm{sccm}$ and adding $\mathrm{Cl}_{2}$ of $6 \mathrm{sccm}$ with the same RF power and working pressure as the first step. He and Ar gas were maintained with flow amounts of $50 \mathrm{sccm}$ and $20 \mathrm{sccm}$, and the etching time for second step was controlled at $20 \mathrm{~s}, 30 \mathrm{~s}$, and $40 \mathrm{~s}$ for $40 \mathrm{~nm}$, $60 \mathrm{~nm}$, and $80 \mathrm{~nm}$ of target etching thickness, respectively. The etch selectivity of the photoresist used in this experiment, $\mathrm{S} 1805$, versus Al was higher than 1:1 and therefore the PR on top of the $\mathrm{Al}$ pattern was removed using a PR-remover after the etching. 

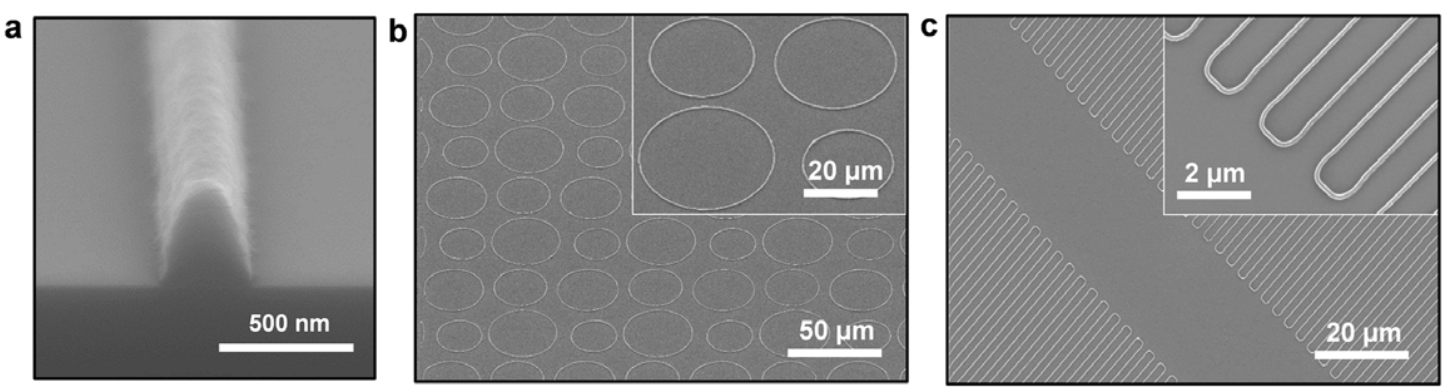

Figure 2. Cross sectional SEM image of fabricated PR patterns by phase lithography (a), by circular phase mask (b), and by a rectangular phase mask (c).

\section{Results and discussion}

This roller phase lithography method combines the advantages of three lithographic techniques, near-field optical lithography (phase-shift lithography), soft lithography, and roll to roll-type patterning technology, and demonstrates the feasibility of fabrication of nanostructures over large areas. In this proof-of-principle experiment, photoresist (PR) coating and development were performed manually. Traditionally, PDMS elastomer material has been used to make the phase mask, because mask patterns can be easily replicated from a master. The elastomeric material can also provide conformal contact to the substrate. The previous approach took advantage of such properties and the patterning was performed without much applied force [12]. In our setup, we intentionally applied force to ensure conformal contacts between the mask and the photoresist layer across the entire width of the quartz cylinder. When a PDMS phase mask was used, we found that the elastomer material and the phase-shift patterns could be deformed by the applied force. Because maintaining a proper pattern height is critical in phase lithography to ensure a $\pi$-phase shift at the resist layer, a phase mask made with low elastic modulus material (e.g. PDMS $\sim 2 \mathrm{MPa}$ ) could not produce reliable results in our roller lithography system. To prevent deformation of the phase mask, we used PUA material instead to fabricate a flexible phase mask. Cured PUA has an elastic modulus of $320 \mathrm{MPa}$, which is two orders of magnitude higher than that of PDMS [17], sufficient to maintain the pattern height under the applied force in the dynamic rolling process.

Figure 2 shows representative line and circular patterns obtained by the phase-shift roll-lithography method. The phase masks used to produce these patterns have raised rectangles and circular disk patterns with plateau heights of $\sim 400 \mathrm{~nm}$. As expected, the resultant patterns on the PR are produced from the edges of the plateaus only due to the $\pi$-phase shift and the resultant zero light intensity. The SEM image in figure 2(a) represents fabricated PR line patterns from rectangular-shaped phase mask patterns. The lines have a trapezoid cross section with sub-100 nm width at the top. As shown in figures 2(b) and (c), various shapes of patterns can be applied that lead to corresponding looped line patterns. Importantly, due to the improved control of the pattern height by the PUA phase mask and the collimated UV light exposure used in our setup, reproducibility of this fabrication method was up to $90 \%$, which represents a remarkable improvement compared with previous research [15].

As a feasible application, a transparent metal electrode was presented in the form of metal mesh patterns. The demand for transparent electrodes has been soaring due to a wide range of applications in liquid crystal displays (LCDs), touch panels, organic light emitting diodes, and, organic photovoltaic devices. There has been increasing interest in finding alternatives to the most commonly used transparent oxide, ITO, because of chemical and physical limitations and the rising cost of ITO electrodes. For this reason, transparent electrodes, such as the metal grid electrode, have been proposed as good alternatives for ITO electrodes [18, 19].

To apply roller phase lithography to the fabrication of a metal mesh such as an electrode, a particular mask design was needed to make well connected mesh patterns. Theoretically, a perfect checkerboard phase mask, as shown in figure 3(a), can produce the desired mesh pattern. In the actual fabrication of the photomask and silicon master, however, vertices of the developed pattern were rounded in the development process. This could be improved by using a thin PR, but a gap of a few hundred nanometers between vertices of any neighboring squares still exists as shown in figure 3(b). To solve this problem, a new mask was designed. According to the simulation results (by Comsol Multiphysics software) shown in figures 3(c) and (d), leaving a deliberately small gap $(\sim 300 \mathrm{~nm})$ between neighboring structures allowed a connected pattern to be produced. For the simulation of a normal sample of width $600 \mathrm{~nm}$ with $600 \mathrm{~nm}$ spacing, there was a $200 \mathrm{~nm}$ wide unexposed region with zero UV intensity. On the other hand, in the case of the modified structure in figure $3(\mathrm{~d})$, two unexposed spots were merged together to form a $300 \mathrm{~nm}$ wide area. To apply this simulation result to experiment, a modified checkerboard mask was designed with discontinuous squares. A small space $(\sim 300 \mathrm{~nm})$ between the two neighboring squares was obtained by a $5 \times$ reduction projection lithography process as shown in figure 3(e). Figures 3(f) and (g) show the fabricated PUA phase mask, having $30 \mu \mathrm{m}$ size square patterns and narrow gaps near the vertices of the squares. The height of the phase mask patterns was determined by the refractive index of the patterned material and the wavelength of the UV light [20],

$$
h=\lambda /(2 \Delta n)
$$




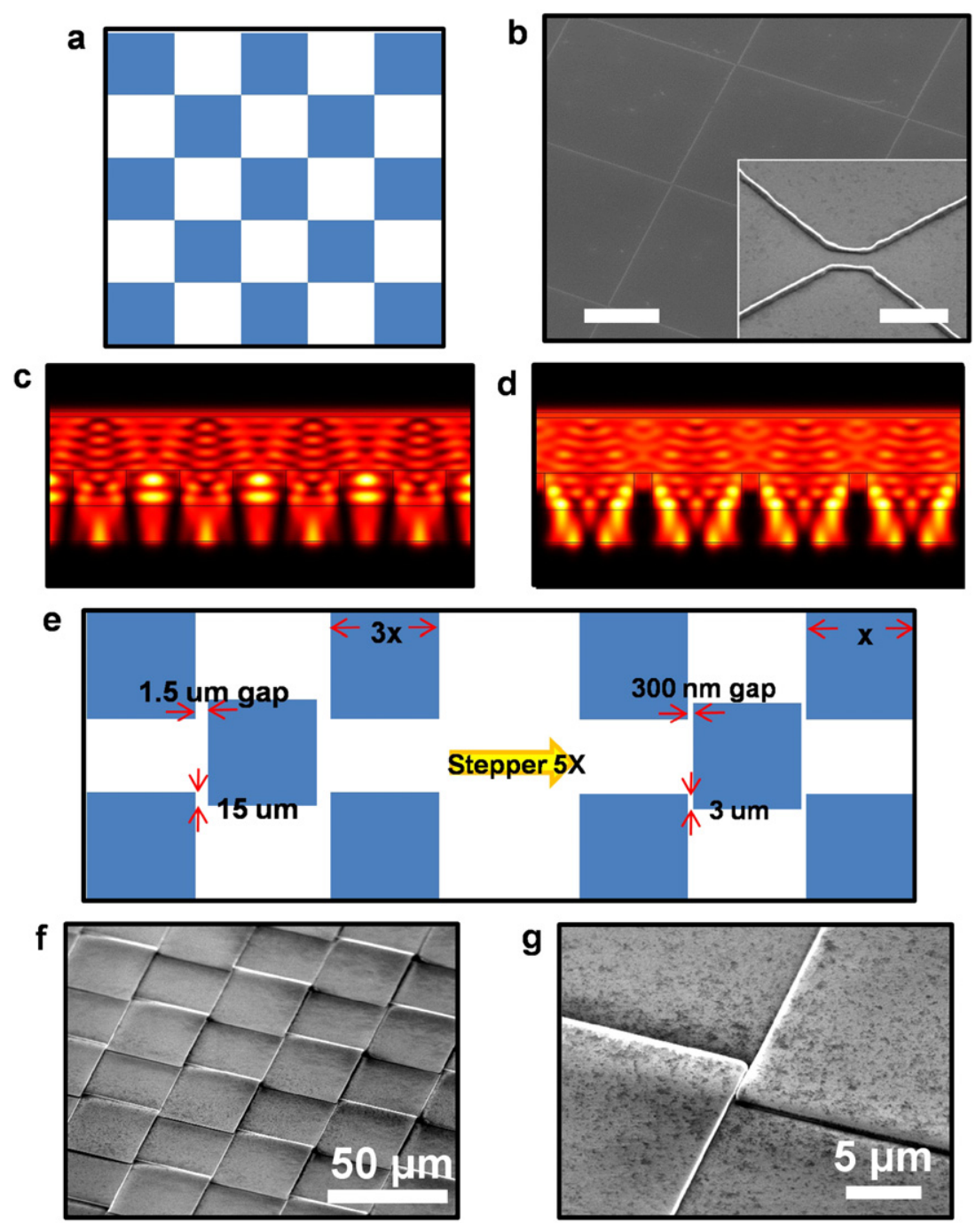

Figure 3. (a) Perfect checkerboard mask design to fabricate a mesh pattern using phase lithography. (b) SEM image of the mesh pattern from the mask design in (a). Scale bars are $100 \mu \mathrm{m}$ and $2 \mu \mathrm{m}$ in the inset. (c) Simulation result of light intensity of mask design (a), and a modified one with a $300 \mathrm{~nm}$ gap between neighboring structures (d). (e) Schematic illustration of mask design for modified checkerboard pattern with a $300 \mathrm{~nm}$ gap between dislocated structures. (f) SEM image of the molded phase mask which was fabricated from the mask design. (g) Magnified SEM image of (f).

where $\lambda$ is wavelength of UV light and $\Delta n$ is the difference in the refraction index between the phase mask material and the surrounding air. In this work, the PUA phase mask is $375 \mathrm{~nm}$ high because PUA has a refractive index of 1.49 when it is cured [21]. Using such masks, PR mesh patterns with about $200 \mathrm{~nm}$ line width can be easily obtained by the roll phase lithography process. By avoiding physical scratching or contamination by an unbaked PR layer, a PUA phase mask can be used more than 500 times.

To make a transparent electrode, the mesh PR patterns are first produced on an Al layer. With further dry etching, aluminum mesh patterns could be fabricated on a glass substrate. Figure 4 shows two $\mathrm{Al}$ mesh patterns of different periods: $10 \mu \mathrm{m}$ (a) and $30 \mu \mathrm{m}$ (b). As expected from the simulation, neighboring squares are connected at the corners with thicker line width. Figure 4(c) shows a zoom-in view of the connected section, about $360 \mathrm{~nm}$ wide, which is about twice the single line width of $170 \mathrm{~nm}$. The fabricated $\mathrm{Al}$ mesh sample was shown in figure 4(d). Because its transparency is about $90 \%$, it looks almost transparent to the naked eye. This fact can be supported by simple calculation of the opening area of the metal mesh patterns: $95 \%$ and $98 \%$ of the opening portion for $10 \mu \mathrm{m}$ and $30 \mu \mathrm{m}$ period samples, respectively. Due to the limitations of the size of the light source and the stroke of the linear motion system in this first prototype, the patterned area was limited to an area of $1 \mathrm{~cm} \times 1 \mathrm{~cm}$. In principle roll phase lithography can be expanded to very large area fabrication, such as twelfth generation LCD panel fabrication $(3350 \mathrm{~mm} \times 3950 \mathrm{~mm})$. Figure 5 shows the experimental data obtained for various conditions of exposure intensity and developing time. With weak UV light, an inverted pattern was observed as reported previously [14]. The SEM images on the second column suggest that the developing process proceeds from the connected part to the center of the squares. Therefore, the proper development time 

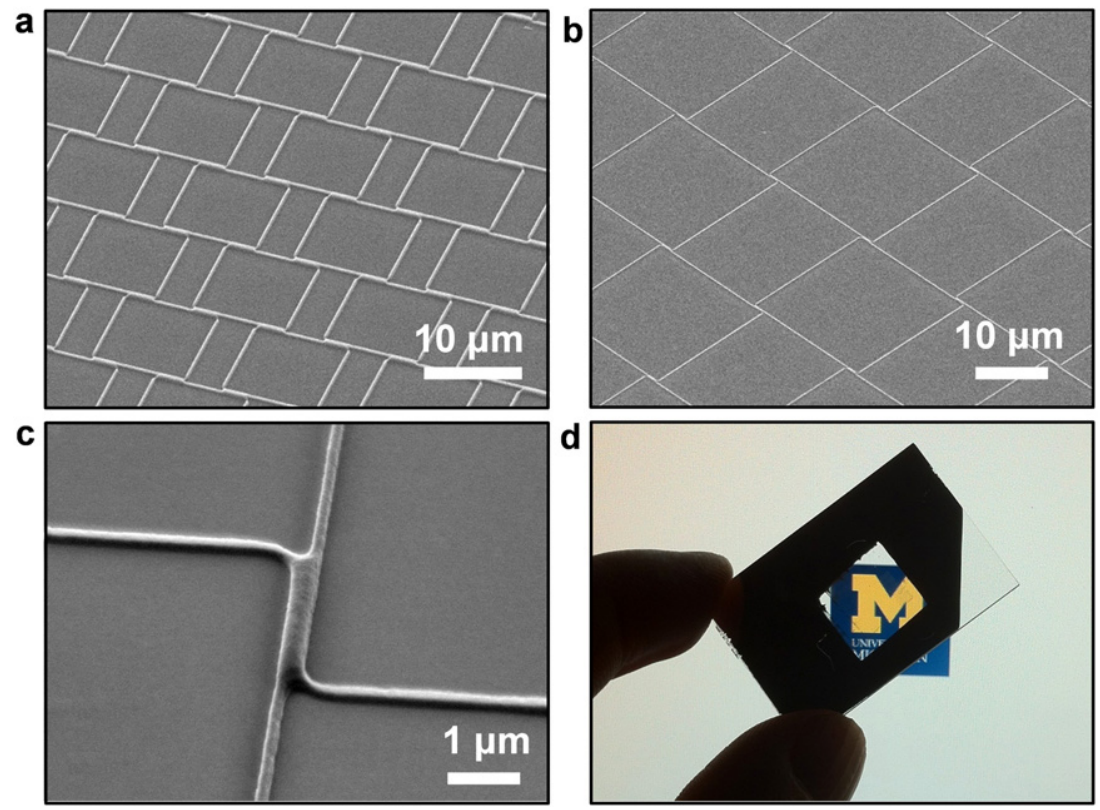

Figure 4. SEM image showing a well connected $\mathrm{Al}$ mesh pattern with $10 \mu \mathrm{m}$ period (a) and with $30 \mu \mathrm{m}$ periodic $\mathrm{Al}$ mesh pattern (b). (c) Magnified image of the connected part between the two rectangular units. (d) Photo of a large area $\left(100 \mathrm{~mm}^{2}\right)$ transparent $\mathrm{Al}$ mesh electrode.

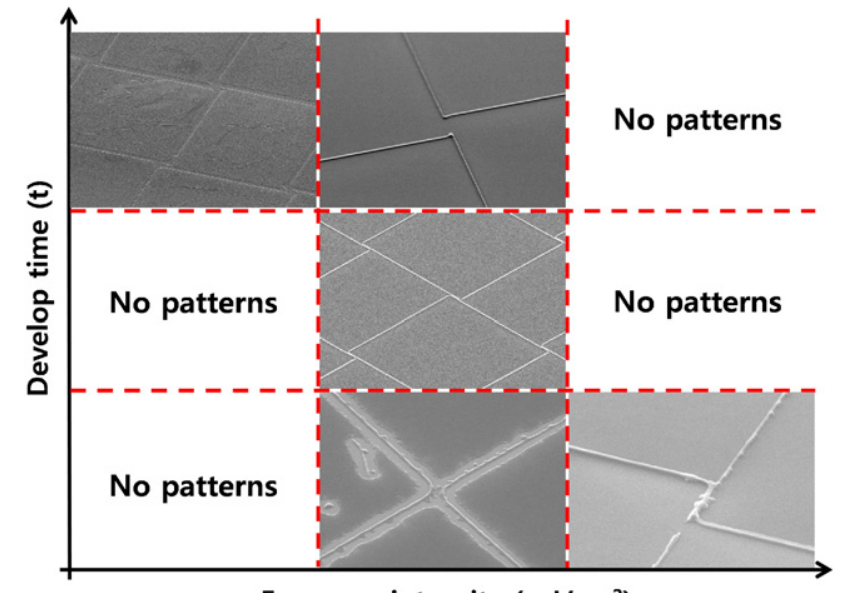

Exposure intensity $\left(\mathrm{mJ} / \mathrm{cm}^{2}\right)$

Figure 5. Diagram showing the effect of a wide range of exposure intensities and development times. The range of $50-75 \mathrm{~s}$ for the development time and $25-45 \mathrm{~mJ} \mathrm{~cm}^{-2}$ shows optimum condition for fabrication. Weak intensity led to a reversed pattern compared with the target pattern, too high an exposure made the patterns disappear. With increasing development time, the exposed sample was developed and erased sequentially from connected parts to the center of the squares.

is one of the most important experimental parameters to obtain well connected mesh patterns.

For a performance test of the transparent metal electrode, the transmittance and sheet resistance of six different samples were measured. As shown in figure 6(a), the transmittance of a mesh pattern is governed by its period while there are no notable differences according to thickness. Regardless of thickness, $10 \mu \mathrm{m}$ periodic patterns have around $85 \%$ transmittance and $30 \mu \mathrm{m}$ periodic patterns have $92 \%$. These values are slightly smaller than those calculated based on opening area. Small defects occurring during the development and etching steps are probably responsible for the reduction of the transmittance. As a reference, the transmittance of commonly used ITO glass is about $85 \%$ (shown as a red solid line in figure 6(a)). Sheet resistance for each case was calculated using Ohm's law and it is expressed by the blue bars in figure 6(b). From the calculated values, it can be recognized that sheet resistance is proportional to the period and the inverse of metal thickness. To measure the sheet resistance, metal pads were deposited on each metal mesh pattern with two different distances (100 and $200 \mu \mathrm{m}$ ). By solving the simultaneous equation which can eliminate the term of contact resistance, sheet resistance of the mesh pattern was measured and those values are shown in figure 6(b) with red bars. A certain amount of mismatch between the measured and calculated values are probably resulted from a few disconnections of metal lines or the change in the conductivity of the metal lines due to rough edges. For six samples, the sheet resistance was measured in the range of $57-195 \Omega / \square$. In comparison with typical sheet resistance of a $100 \mathrm{~nm}$ thick ITO, 20-80 $\Omega / \square$, these metal mesh electrodes can be a potential replacement for ITO in certain applications, e.g. in touch panels.

\section{Conclusion}

In summary, we have presented a simple method for fabricating sub-wavelength scale patterns by a continuous phase lithography setup using a roll-type phase mask. The residual layer-free sub-wavelength scale pattern was achieved on a large area, thereby addressing some of the limitations of photolithography and roll to roll imprinting. The large 

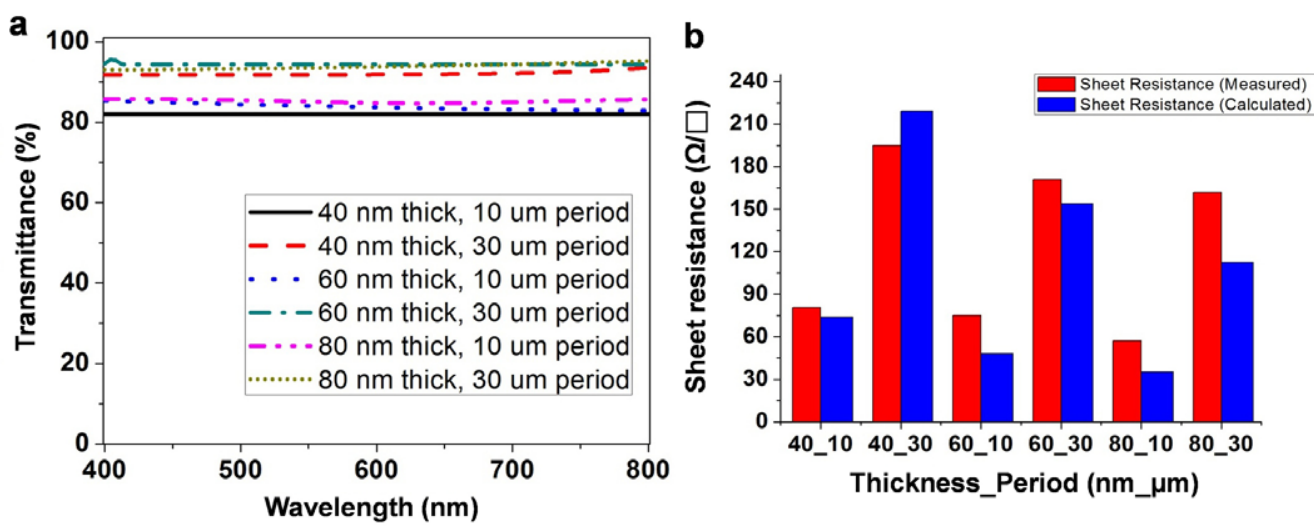

Figure 6. (a) Transmittance of various transparent electrode samples with three different thicknesses and two kinds of periods. The red line indicates the transmittance of ITO glass. (b) Measured sheet resistance values (red) for fabricated samples and calculated values (blue) for them. The black line indicates the sheet resistance of ITO glass.

area capability and the diversity of the substrates that can be used in the process make it attractive for large area device applications. We also proposed a method to produce metal mesh patterns as a transparent electrode. The mass production method for transparent metal electrodes, which are a promising alternative to the ITO electrode, has a scientific and industrial significance. The new process can allow large area and cost effective fabrication of metal mesh electrodes.

\section{Acknowledgments}

This work was supported in part by the National Science Foundation. Electron microscopy analysis was performed in the Electron Microbeam Analysis Laboratory (EMAL), and nanofabrication was performed in the Lurie Nanofabrication Facility (LNF) at the University of Michigan.

\section{References}

[1] Jeong H E, Kwak M K, Park C I and Suh K Y 2009 J. Colloid Interface Sci. 339202

[2] Lau K K S, Bico J, Teo K B K, Chhowalla M, Amaratunga G A J, Milne W I, McKinley G H and Gleason K K 2003 Nano Lett. 31701

[3] Kwak M K, Pang C, Jeong H E, Kim H N, Yoon H, Jung H S and Suh K Y 2011 Adv. Funct. Mater. 213606

[4] Garnett E and Yang P 2010 Nano Lett. 101082

[5] Kang M G, Xu T, Park H J, Luo X and Guo L J 2010 Adv. Mater. 224378
[6] Ahn S W, Lee K D, Kim J S, Kim S H, Park J D, Lee S H and Yoon P W 2005 Nanotechnology 161874

[7] Kwak M K, Kim T, Kim P, Lee H H and Suh K Y 2009 Small 5928

[8] Chanda D, Shigeta K, Gupta S, Cain T, Carlson A, Mihi A, Baca A J, Bogart G R, Braun P and Rogers J A 2011 Nature Nanotechnol. 6402

[9] Shalaev V M 2007 Nature Photon. 141

[10] Ahn S H and Guo L J 2008 Adv. Mater. 202044

[11] Ahn S H, Kim J S and Guo L 2007 J. Vac. Sci. Technol. B 252388

[12] Kobrin B, Barnard E, Brongersma M, Kwak M K and Guo L J 2012 Rolling mask nanolithography - the pathway to large area and low cost nanofabrication SPIE Photonics West (San Francisco, Jan.) also see Rolith.com

[13] Henzie J, Barton J E, Stender C L and Odom T W 2006 Acc. Chem. Res. 39249

[14] Lee T W, Jeon S, Maria J, Zaumseil J, Hsu J W P and Rogers J A 2005 Adv. Funct. Mater. 151435

[15] Rogers J A, Paul K E, Jackman R J and Whitesides G M 1997 Appl. Phys. Lett. 702658

[16] Choi S J, Kim H N, Bae W G and Suh K Y 2011 J. Mater. Chem. 2114325

[17] Yoo P J, Choi S J, Kim J H, Suh D, Baek S J, Kim T W and Lee H H 2004 Chem. Mater. 165000

[18] Kang M G and Guo L J 2007 Adv. Mater. 19 1391-6

[19] Kang M G, Kim M S, Kim J and Guo L J 2008 Adv. Mater. 204408

[20] Rogers J A, Paul K E, Jackman R J and Whitesides G M 1998 J. Vac. Sci. Technol. B 16 59-68

[21] Minuta Technology Co., Ltd webpage, products property 'MINS-311RM' www.minuta.co.kr/products/products_ mold_template.html 